\title{
THE EFFECT OF EXOGENOUS PHOSPHOROUS APPLICATION ON GROWTH, YIELD, QUALITY AND NET RETURNS OF UPLAND COTTON (GOSSIPIUM HIRSUTUM L.)
}

\author{
ALI, H. - AHMAD, A. - HUSSAIN, S. * \\ Department of Agronomy, Faculty of Agricultural Sciences and Technology, Bahauddin \\ Zakariya University, Multan, Pakistan \\ *Corresponding author \\ e-mail: shabirhussain@bzu.edu.pk \\ (Received $2^{\text {nd }}$ Jul 2019; accepted $25^{\text {th }}$ Oct 2019)
}

\begin{abstract}
Cotton crop is grown in China, Brazil, Pakistan, India and in the USA. It is a major global resource in textile production. Phosphorus has major role in the growth and yield of plants facilitating higher crop production. Phosphorous is involved in plant growth and opening of flower bud while low nitrogen and magnesium absorption inhibits plant growth. Higher input cost of fertilizers and numerous other yield limiting factors are major problems for farmers. To mitigate the problem, present study was carried out at the Central Cotton Research Institute (CCRI), Multan, Pakistan. The experimental treatments comprised of three cultivars viz. $\mathrm{V}_{1}=\mathrm{MNH}-886, \mathrm{~V}_{2}=\mathrm{CIM}-616$ and $\mathrm{V}_{3}=$ FH-LALAZAR and seven levels of Phosphorous viz. To $=$ Zero $\mathrm{kg} \mathrm{ha}^{-1}, \mathrm{~T}_{1}=50 \mathrm{~kg} \mathrm{ha}^{-1}, \mathrm{~T}_{2}=50 \mathrm{~kg} \mathrm{ha}^{-1}+$ foliar ( $2 \%$ TSP solution), $\mathrm{T}_{3}=75 \mathrm{~kg}$ $\mathrm{ha}^{-1}, \mathrm{~T}_{4}=75 \mathrm{~kg} \mathrm{ha}^{-1}+$ foliar ( $2 \%$ solution of Triple super phosphate), $\mathrm{T}_{5}=100 \mathrm{~kg} \mathrm{ha}^{-1}+$ seed application (5\% solution of triple super phosphate), $\mathrm{T}_{6}=100 \mathrm{~kg} \mathrm{ha}^{-1}$ phosphorous (with seed application of a $5 \%$ solution of triple super phosphate, and foliar application of a $2 \%$ solution). Different genotypes significantly differed in various growth, yield and quality attributes. In this regard, the cultivar FH- Lalazar produced the highest plant height $(152.50 \mathrm{~cm})$, lint yield $\left(50.58 \mathrm{~kg} \mathrm{ha}^{-1}\right)$, seed cotton harvest $(27 \%)$, ginning out turn $(0.02 \%)$, crop growth rate $\left(4.79 \mathrm{~kg} \mathrm{ha}^{-1}\right)$, fiber uniformity (45.50), fiber strength (30.16 tppsi) and micronair (4.72 $\mu \mathrm{g} / \mathrm{inch})$. CIM-616 showed the maximum number of bolls/plant (28.91), leaf area index $\left(0.65 \mathrm{~kg} \mathrm{ha}^{-1}\right)$, boll weight $(2.98 \mathrm{~g})$, and staple length $(20.19 \mathrm{~mm})$. In conclusion, cultivar FH-LZ using $100 \mathrm{~kg} \mathrm{ha}^{-1}$ phosphorous (with seed application of a 5\% solution of triple super phosphate, and foliar application of a $2 \%$ solution) produced higher yield, ginning out turn, seed cotton harvest, crop growth rate and fiber uniformity with lower input cost and attained the maximum output with higher crop production.
\end{abstract}

Keywords: yields, transgenic cotton, fiber quality, foliar application, phosphorous concentration

\section{Introduction}

Cotton is a commercial crop for its excellent quality as fiber and oil so it is grown in whole regions of the world (Zhang et al., 2012). Lately, cotton has been grown in a region of 2907 thousand hectares while the ranks of production is 13.2 million bales (Govt. of Pakistan, 2018). During flowering and boll opening stage the continuous rain may possibly cause the reduction of pollination phenomenon and ultimately minimize fiber quality. Therefore, heavy rainfall causes flower buds and young bolls to fall during flowering (Gaikwad, 2018).

Nutritional quality of crops can be enhanced and may vary depending upon the soil conditions as well as genetic make-up of genotypes (Zhang et al., 2012; Ahmad et al., 2019). However, optimum dose of macro-nutrients as well as micronutrients and their proper application maximized crop production (Vance et al., 2003). Reduction of phosphorous availability resulted in minimizing the cotton plant growth. Reduction of phosphorus causes the minimization of different traits i.e. reduced flowering, imbalanced pollination, boll weight and boll size. Moreover, low phosphorus in cotton crop inhibits 
dark green leaves and flower bud necrosis. Lower phosphorous availability in cotton crop also affects the development of flower buds and also minimized the absorption of nitrogen resulting in inhibited cell growth and their expansion (Dohary et al., 2004).

Phosphorous is taken up by the roots. So among all of this many factors which affect the cotton response to phosphorous the most is the type of soil. The acute amount of phosphorous is a main issue of actual concentration of phosphorous, which is provided at the time of cotton growth (Crozier et al., 2004). Numerous traits i.e. early phosphorous accumulation and lint yield positively respond to phosphorous in calcareous soils (Bronson et al., 2003). However, the reduction of phosphorous may possibly minimize the nitrogen level. Due to the indeterminate type of growth and deep-growing root system of cotton crop phosphorous concentration availability is low (Zhou et al., 2011).

Several nutrients were applied through foliar spray to leaves that may prevent their senescence and decline in photosynthesis allowing photosynthesis and carbon fixation to be prolonged thus increasing the yield of cotton (Bronson et al., 2001). Those resistant cultivars were selected that had greater capability to tolerate unfavorable conditions including nutritional stress which results in higher yield of crop (Zambrosi et al., 2012). The efficiency of phosphorus cultivars having the ability to grow well and produce the maximum yield in soil with the minimum phosphorous (Fageria et al., 2010). Thus, effiicnet application of phosphorus was recorded in several agronomic crops. (Zambrosi et al., 2012; Fageria and Moraes, 2013).

Foliar application and seed treatments are successfully used in application of several nutrients especially phosphorus. Among all the nutrient application methods, foliar application is considered as one of the most suitable method and it is also helpful for proper use of nutrients in plants (Girma et al., 2007). Furthermore, combination of foliar application and seed treatment results in efficient use of phosphorus to plants and ultimately enhanced cotton production. Application of phosphorus to all other agronomic crops is very common; however, its use in cotton is very limited. Therefore, current study is more helpful for cotton growers to enhance production through foliar application of phosphorus (Verma et al., 2018). The optimum dose of phosphorus and its timely application drastically enhance cotton crop production all over the globe (Araújo et al., 2012). So, the purpose of this current study was to demonstrate the optimum application of phosphorus for various cotton cultivars.

\section{Materials and methods}

\section{Experimental details}

Current study was conducted in the research farm of Central Cotton Research Institute (CCRI) Multan, Pakistan to mitigate various cultivars of cotton with various foliar application of phosphorus during 2015-2016. The soil at the Research Farm was silt loam and its organic content was $0.86 \%$, Nitrogen concentration was $0.09 \%$, Phosphorous concentration was $12.5 \mathrm{ppm}$, Sand was $16 \%$, Silt was 57\%, Clay was 27\%. Experimental treatments comprised of three cotton cultivars viz. $\mathrm{V}_{1}=\mathrm{MNH}-886, \mathrm{~V}_{2}=\mathrm{CIM}-616$ and $\mathrm{V}_{3}=$ FH-LALAZAR and seven phosphorus levels viz. To $=$ Zero, $\mathrm{T}_{1}=50 \mathrm{~kg} \mathrm{ha}^{-1}, \mathrm{~T}_{2}=50$ $\mathrm{kg} \mathrm{ha}^{-1}+$ foliar (2\% TSPsolution), $\mathrm{T}_{3}=75 \mathrm{kgha}^{-1}, \mathrm{~T}_{4}=75 \mathrm{~kg} \mathrm{ha}^{-1}+$ application of foliar ( $2 \%$ solution of triple super phosphate), $\mathrm{T}_{5}=100 \mathrm{~kg} \mathrm{ha}^{-1}+$ seed application (5\%solution of triple super phosphate), $\mathrm{T}_{6}=100 \mathrm{~kg} \mathrm{ha}^{-1}$ phosphorous (with seed application of a $5 \%$ solution of triple super phosphate, and foliar application of a $2 \%$ solution). One meter area was marked from the center of the plot from where sampling for various parameters 
was done. CCRI lies at a latitude of $30^{\circ}$, and at a longitude of $71^{\circ}$, the altitude is 125 in the cotton belt of Punjab. After the preparation of seed bed the sowing was carried out with cotton Rabi drill on the $28^{\text {th }}$ May, 2015 and on the $31^{\text {st }}$ May, 2016 respectively. Net plot size was $6.1 \mathrm{~m} \times 9.0 \mathrm{~m}=54.9 \mathrm{~m}^{2}$ with a sowing rate of $110 \mathrm{~g} / \mathrm{plot}$. Randomized Complete Block Design (RCBD) was applied along three replications. Urea fertilizers (36 $\mathrm{kg} \mathrm{ha}^{-1}$ ) were applied as per standard rule according to crop requirement, and seven phosphorous levels were used and TSP as a source were also applied. Whole of phosphorous according to treatments done on sowing time and application of nitrogen done on three intervals as per standard roles at both years. Pendimethaline (herbicide) was applied at the rate of $82.5 \mathrm{~g} \mathrm{ha}^{-1}$ to control weeds. For the chewing and sucking insect pest different products such as Nitenpyren, Diafenthoran, Triazophos were applied and Leuforan Irrigation was performed as crop needed. Harvesting was done from 05-11-2015 and 13-11-2016 and picking interval was two weeks.

\section{Observations}

\section{Agronomic traits}

Five plants were randomly chosen from every plot and the height of the plant was observed with a measuring tape in between about the base of the plant to the terminal bud. Same plants were used to measure no. of bolls/plant. One hundred fully opened bolls were selected for picking to get a hundred boll weight. Yield of cotton was calculated after picking by same plot, after this the bolls were weighed to get the yield $\left(\mathrm{kg} \mathrm{ha}^{-1}\right)$. Ginning out turn (\%) was calculated by the following formula: mass of cotton lint/mass of seed cotton x 100 (Baloch et al., 2014).

\section{Allometry traits}

Leaf area of each plant was measured with a leaf area meter (CI-203, LCi Bio Scientific, USA). Leaf area index (LAI) was calculated according to the method of Hunt (1978) using the following formula: LAI = Leaf Area/Ground Area Leaf. Area duration was recorded simultaneously by $\mathrm{LAI}_{1}$ and $\mathrm{LAI}_{2}$ with leaf area indices at times $\mathrm{T}_{1}$ and $\mathrm{T}_{2}$ and $\mathrm{LAD}($ days $)=\left(\mathrm{LAI}_{1}+\mathrm{LAI}_{2}\right) \times\left(\mathrm{T}_{2}-\mathrm{T}_{1}\right) / 2$ (Hassan et al., 2016). Crop growth rate was measured by collecting weighed samples of 10,30, $60 \mathrm{~g}$ of green leaves, stalks and bolls collected by same treatment at various stages of plant growth, which then were dried in the oven at $80{ }^{\circ} \mathrm{C}$. Crop growth rate was calculated by using the formula of Hunt (1978).

\section{Quality traits}

Quality traits, cotton seed were ginned in an electrically powered ginning machine to separate lint from seed followed by cleaning of those samples. Each sample was acclimatized at an optimum temperature of $20^{\circ} \mathrm{C}$ with $65 \%$ relative humidity level in an air conditioned chamber. The level of humidity was measured by a humidifier (HIV900) at the humidity level of $8.5 \%$ Tisarum et al. (2019). Fiber strength is calculated by breaking the fibers held between clamp jaws. It is mentioned as $\mathrm{g} / \mathrm{tex}$, which is the force used to break bundle (Apel and Hirt2004)

\section{Data analysis}

Data was analyzed by using Statistix 8.1 (Tallahassee Florida, USA) on computer. The means of treatment were separately analyzed by LSD @ 5\% level of probability. 
Pearson's correlation was constructed through software XLSTAT, 2017(Anjum et al., 2018; Ahmad and Anjum, 2018). Whereas economic analysis was performed as determined by Ahmad et al. (2016). Climatic data of experimental site was evaluated as described (Pakistan Metrological Department, 2016).

\section{Results}

Different cotton cultivars and yield related traits were significantly differed in the current study. Significantly higher plant height $(60.03$ inch) was recorded from Cultivar FH-Lalazar and the lowest height of plant (57.74 inch) was measured from MNH-886. The treatment $100 \mathrm{~kg} \mathrm{ha}^{-1}+$ seed application (5\% solution of triple super phosphate) (61.02 inch), under $100 \mathrm{~kg} \mathrm{ha}^{-1}$ phosphorous (with seed application of a 5\% solution of triple super phosphate, and foliar application of a $2 \%$ solution) resulted in a significantly higher plant height (61.93 inch), while the minimum plant height was calculated from control (56.38 inch). Cultivars and phosphorus levels interaction showing significantly higher plant height (61.81 inch) was recorded from FH-LZ with $75 \mathrm{~kg} \mathrm{ha}^{-1}+$ foliar (2\% TSP solution), followed by the plant height (63.38) from FH-LZ with100 kg ha ${ }^{-1}+$ seed treatment (5\% TSP solution), followed by the plant height $\left(64.17\right.$ inch) from FH-LZ under $100 \mathrm{~kg} \mathrm{ha}^{-1}$ phosphorous (with seed application of a $5 \%$ solution of triple super phosphate, and foliar application of a $2 \%$ solution), while the minimum (55.24 inch) was recorded from MNH-886 with Zero kg ha-1 (Table 1). The higher the no. of bolls/plant (28.91) recorded from CIM-616, the lower the no. of bolls/plant (24.50) occurred from cultivar MNH-886. From treatment $100 \mathrm{~kg} \mathrm{ha}^{-1}$ + seed solution (5\% solution of triple super phosphate) (29.11) under $100 \mathrm{~kg} \mathrm{ha}^{-1}$ phosphorous (with seed application of a 5\% solution of triple super phosphate, and foliar application of a $2 \%$ solution) higher no. of bolls/plant were found (29.65), the minimum in no. of bolls/plant was found in control (23.77). Regarding the level of phosphorous and cultivars interaction the highest no. of bolls/plant (35.33) occurred from CIM-612 under $100 \mathrm{~kg} \mathrm{ha}^{-1}$ phosphorous (with seed application of a $5 \%$ solution of triple super phosphate, and foliar application of a $2 \%$ solution), followed by (33.33) from CIM-612 with $100 \mathrm{~kg} \mathrm{ha}$ ${ }^{1}+$ seed treatment (5\% TSP solution). So the minimum no. of bolls/plant (22.00) was present in MNH-886 with control (Table 1).

The maximum boll weight (2.98 g) was measured from CIM-616, the lowest $(1.21 \mathrm{~g})$ from MNH-886. Concerning the phosphorus applications, the treatment $75 \mathrm{~kg} \mathrm{ha}^{-1}(2.26 \mathrm{~g})$, $75 \mathrm{~kg} \mathrm{ha}^{-1}+$ foliar (2\% TSP solution), $(2.27 \mathrm{~g}), 100 \mathrm{~kg} \mathrm{ha}^{-1}+$ application of seed treatment (5\% TSP solution) (2.29 g) under $100 \mathrm{~kg} \mathrm{ha}^{-1}$ phosphorous (with seed application of a 5\% solution of triple super phosphate, and foliar application of a $2 \%$ solution) (2.45 g) increased boll weight, and a decrease of boll weight was measured from control $(1.60 \mathrm{~g})$. Regarding the levels of phosphorous and cultivars maximum weight of boll (3.20 g) was calculated from CIM-616 by50 kg ha-1, followed by ( $3.22 \mathrm{~g}$ ) from CIM-616 with $50 \mathrm{~kg} \mathrm{ha}^{-1}$ + foliar (2\% TSP solution), followed by (3.30 g) from CIM-616 with $75 \mathrm{~kg} \mathrm{ha}^{-1}$, followed by (3.32 g) from CIM-616 with $75 \mathrm{~kg} \mathrm{ha}^{-1}+$ foliar (2\%TSPsolution), followed by (3.30 $\mathrm{g}$ ) from CIM-616 with $100 \mathrm{~kg} \mathrm{ha}^{-1}+$ seed treatment (5\% TSPsolution) and (3.32 g) from CIM 616 under $100 \mathrm{~kg} \mathrm{ha}^{-1}$ phosphorous (with seed application of a $5 \%$ solution of triple super phosphate, and foliar application of a $2 \%$ solution) (Table 1). The highest cotton yield was found from FH-Lalazar (2809.3 $\left.\mathrm{kg} \mathrm{ha}^{-1}\right)$, while the lowest was $\left(1905.6 \mathrm{~kg} \mathrm{ha}^{-1}\right)$ from MNH886. Level of application $75 \mathrm{~kg} \mathrm{ha}^{-1}\left(2638.00 \mathrm{~kg} \mathrm{ha}^{-1}\right), 75 \mathrm{~kg} \mathrm{ha}^{-1}+$ foliar (2\% TSP solution), (2651.00 kg ha-1), $100 \mathrm{~kg} \mathrm{ha}^{-1}+$ seed treatment (5\% TSP solution) (2763.9 kg ha${ }^{1}$ ), under $100 \mathrm{~kg} \mathrm{ha}^{-1}$ phosphorous (with seed application of a 5\% solution of triple super 
phosphate, and foliar application of a $2 \%$ solution) (2769.9 $\left.\mathrm{kg} \mathrm{ha}^{-1}\right)$ showed statistically higher yield of cotton, the minimum cotton yield $\left(1941.1 \mathrm{~kg} \mathrm{ha}^{-1}\right)$ was determined from control, and $50 \mathrm{~kg} \mathrm{ha}^{-1}\left(2002.3 \mathrm{~kg} \mathrm{ha}^{-1}\right)$ and $50 \mathrm{~kg} \mathrm{ha}^{-1}+$ foliar (2\% TSP solution) $\left(2004.3 \mathrm{~kg} \mathrm{ha}^{-1}\right)$. Cultivars with levels of phosphorus interaction gave the highest cotton yield (3776.3 $\left.\mathrm{kg} \mathrm{ha}^{-1}\right)$ measured from FH-Lalazar with $75 \mathrm{~kg} \mathrm{ha}^{-1},\left(3976.3 \mathrm{~kg} \mathrm{ha}^{-1}\right)$ from cultivar FH-LZ with75 $\mathrm{kg} \mathrm{ha}^{-1}+$ foliar (2\% TSP solution), followed by (3844.00 $\mathrm{kg} \mathrm{ha}^{-1}$ ) from cultivar FH-Lalazar with100 $\mathrm{kg} \mathrm{ha}^{-1}+$ seed treatment (5\% TSP solution) and (4044.0 $\mathrm{kg} \mathrm{ha}^{-1}$ ) from FH-LZ under $100 \mathrm{~kg} \mathrm{ha}^{-1}$ phosphorous (with seed application of a 5\% solution of triple super phosphate, and foliar application of a $2 \%$ solution); while lower cotton yield (1561.7 $\mathrm{kg} \mathrm{ha}^{-1}$ ) was measured from FH-LZ with Zero kg ha-1 (Table 1).

Table 1. Influence of phosphorous application methods on plant height, number of bolls/plant, boll weight, seed cotton yield of cotton cultivars

\begin{tabular}{|c|c|c|c|c|c|c|c|c|c|}
\hline Variables & Cultivars & Control & $50 \mathrm{~kg} \mathrm{ha}^{-1}$ & $50+$ foliar & $75 \mathrm{~kg} \mathrm{ha}^{-1}$ & 75+foliar & $100+$ seed treat & $100+$ s+fol & Means \\
\hline \multirow{4}{*}{ Plant height (inch) } & MNH-886 & $55.24 \mathrm{f}$ & $58.53 \mathrm{~d}$ & $58.53 \mathrm{~d}$ & $58.53 \mathrm{~d}$ & $59.84 \mathrm{bc}$ & $60.23 b c$ & $60.62 b c$ & $57.74 \mathrm{c}$ \\
\hline & CIM-616 & $56.95 \mathrm{e}$ & $58.66 \mathrm{~d}$ & $58.66 \mathrm{~d}$ & $59.05 \mathrm{~d}$ & $59.44 \mathrm{~cd}$ & $59.44 \mathrm{~cd}$ & $60.23 \mathrm{~cd}$ & $58.53 \mathrm{~b}$ \\
\hline & FH-LZ & $56.95 \mathrm{e}$ & $58.79 \mathrm{~d}$ & $58.79 \mathrm{~d}$ & $61.02 \mathrm{~b}$ & $62.99 \mathrm{a}$ & $63.38 \mathrm{a}$ & $63.77 \mathrm{a}$ & $60.03 \mathrm{a}$ \\
\hline & Means & $56.38 \mathrm{~d}$ & $58.13 \mathrm{c}$ & $58.62 \mathrm{c}$ & $59.53 \mathrm{~b}$ & $60.07 \mathrm{~b}$ & $61.02 \mathrm{a}$ & $61.93 \mathrm{a}$ & - \\
\hline $\operatorname{LSD}(\mathrm{P} \leq 0.05)$ & \multicolumn{6}{|c|}{ CULTIVAR $(\mathrm{C})=1.23$} & \multicolumn{3}{|c|}{$\mathrm{C} \times \mathrm{P}=3.57$} \\
\hline \multirow{4}{*}{ Number of bolls/plant } & MNH-886 & $22.00 \mathrm{f}$ & $23.66 \mathrm{ef}$ & $25.66 \mathrm{ef}$ & $25.66 \mathrm{cde}$ & $27.66 \mathrm{cde}$ & $27.00 \mathrm{bc}$ & $29.00 \mathrm{bc}$ & $24.50 \mathrm{c}$ \\
\hline & CIM-616 & $25.33 \mathrm{cde}$ & $27.66 \mathrm{bc}$ & $29.66 \mathrm{bc}$ & $29.33 b$ & $31.33 \mathrm{~b}$ & $33.33 \mathrm{a}$ & $35.33 \mathrm{a}$ & $28.91 \mathrm{a}$ \\
\hline & FH-LZ & $24.00 \mathrm{def}$ & 25.66 cde & $25.66 \mathrm{cde}$ & 26.66bcde & 28.66 bcde & $27.00 \mathrm{bc}$ & $29.00 \mathrm{bc}$ & $25.83 \mathrm{~b}$ \\
\hline & Means & $23.77 \mathrm{~d}$ & $25.66 \mathrm{c}$ & $25.67 \mathrm{c}$ & $27.11 \mathrm{~b}$ & $28.98 \mathrm{~b}$ & $29.11 \mathrm{a}$ & $29.65 \mathrm{a}$ & - \\
\hline $\operatorname{LSD}(\mathrm{P} \leq 0.05)$ & \multicolumn{6}{|c|}{ CULTIVAR $(\mathrm{C})=1.04$} & \multicolumn{3}{|c|}{$\mathrm{C} \times \mathrm{P}=3.02$} \\
\hline \multirow{4}{*}{ Boll weight (g) } & MNH-886 & $1.03 \mathrm{e}$ & $1.16 \mathrm{e}$ & $1.18 \mathrm{e}$ & $1.33 \mathrm{de}$ & $1.35 \mathrm{de}$ & $1.33 \mathrm{de}$ & $1.35 \mathrm{de}$ & $1.21 \mathrm{c}$ \\
\hline & CIM-616 & $2.13 \mathrm{~b}$ & $3.20 \mathrm{a}$ & $3.22 \mathrm{a}$ & $3.30 \mathrm{a}$ & $3.32 \mathrm{a}$ & $3.30 \mathrm{a}$ & $3.32 \mathrm{a}$ & $2.98 \mathrm{a}$ \\
\hline & FH-LZ & $1.63 \mathrm{~cd}$ & $1.93 \mathrm{bc}$ & $1.95 \mathrm{bc}$ & $2.26 \mathrm{~b}$ & $2.28 \mathrm{~b}$ & $2.23 \mathrm{~b}$ & $2.25 \mathrm{~b}$ & $1.99 \mathrm{~b}$ \\
\hline & Means & $1.60 \mathrm{c}$ & $2.10 \mathrm{~b}$ & $2.13 \mathrm{~b}$ & $2.26 \mathrm{a}$ & $2.27 \mathrm{a}$ & $2.29 \mathrm{a}$ & $2.45 \mathrm{a}$ & - \\
\hline $\mathrm{LSD}(\mathrm{P} \leq 0.05)$ & \multicolumn{6}{|c|}{ CULTIVAR $(\mathrm{C})=0.11$} & \multicolumn{3}{|c|}{$\mathrm{C} \times \mathrm{P}=0.33$} \\
\hline \multirow{4}{*}{ Seed cotton yield $\%$} & MNH-886 & $1776.3 \mathrm{fg}$ & 1844.0efg & 2044.0 efg & $1932.7 \mathrm{def}$ & $2132.7 \mathrm{def}$ & $2069.3 \mathrm{def}$ & 2269.3 def & $1905.6 \mathrm{c}$ \\
\hline & CIM-616 & $2485.3 \mathrm{~b}$ & 2108.0cde & 2308 cde & 2208.0cde & $2408.0 \mathrm{cde}$ & $2378.3 \mathrm{bc}$ & $2578.3 \mathrm{bc}$ & $2294.9 \mathrm{~b}$ \\
\hline & FH-LZ & $1561.7 \mathrm{~g}$ & 2055.0def & $2255.0 \mathrm{def}$ & $3776.3 \mathrm{a}$ & $3976.3 \mathrm{a}$ & $3844.0 \mathrm{a}$ & $4044.0 \mathrm{a}$ & $2809.3 \mathrm{a}$ \\
\hline & Means & $1941.1 \mathrm{~b}$ & $2002.3 \mathrm{~b}$ & $2004.3 \mathrm{~b}$ & $2638.0 \mathrm{a}$ & $2651.0 \mathrm{a}$ & $2763.9 \mathrm{a}$ & $2769.9 \mathrm{a}$ & - \\
\hline $\operatorname{LSD}(\mathrm{P} \leq 0.05)$ & \multicolumn{3}{|c|}{ CULTIVAR $(\mathrm{C})=104.88$} & pho & sphorus lev & $(\mathrm{P})=133$. & \multicolumn{3}{|c|}{$\mathrm{C} \times \mathrm{P}=302.71$} \\
\hline
\end{tabular}

Mean values sharing similar letters are statistically significant at $p=0.05$

Cultivar FH-Lalazar (50.58 $\left.\mathrm{kg} \mathrm{ha}^{-1}\right)$ gave the highest lint yield, the lowest yield of lint was measured (37.33 $\mathrm{kg} \mathrm{ha}^{-1}$ ) from cultivar MNH-886. The treatment $50 \mathrm{~kg} \mathrm{ha}^{-1}$ (44.00), $50 \mathrm{~kg} \mathrm{ha}^{-1}+$ foliar (2\% TSP solution)(2004.3 $\left.\mathrm{kg} \mathrm{ha}^{-1}\right)$. (44.55), $75 \mathrm{~kg} \mathrm{ha}^{-1}$ (44.88), $75 \mathrm{~kg} \mathrm{ha}^{-1}+$ foliar (2\% TSP solution) (45.00), $100 \mathrm{~kg} \mathrm{ha}^{-1}+$ seed application (5\% solution of triple super phosphate) (45.55) under $100 \mathrm{~kg} \mathrm{ha}^{-1}$ phosphorous (with seed application of a 5\% solution of triple super phosphate, and foliar application of a $2 \%$ solution) $\left(45.89 \mathrm{~kg} \mathrm{ha}^{-1}\right)$ resulted in statistically higher lint yield, the minimum lint yield was (40.00 $\left.\mathrm{kg} \mathrm{ha}^{-1}\right)$ obtained from control (Table 2). FH-Lalazar (0.76 kg ha-1) showed statistically higher biological yield, the lowest biological yield was determined from two cultivars MNH-886 (0.69 kg ha $\left.{ }^{-1}\right)$ and CIM-616 $\left(0.67 \mathrm{~kg} \mathrm{ha}^{-1}\right)$. Treatment 100 $\mathrm{kg} \mathrm{ha}^{-1}+$ seed treatment (5\% TSP solution) $\left(0.83 \mathrm{~kg} \mathrm{ha}^{-1}\right)$ under $100 \mathrm{~kg} \mathrm{ha}^{-1}$ phosphorous (with seed application of a 5\% solution of triple super phosphate, and foliar application of a $2 \%$ solution) $\left(0.92 \mathrm{~kg} \mathrm{ha}^{-1}\right)$ showed statistically higher biological 
yield, the minimum biological yield $\left(0.57 \mathrm{~kg} \mathrm{ha}^{-1}\right)$ was obtained from control (Table 2). Higher seed cotton harvest $(27 \%)$ was calculated from FH-LZ, while the minimum seed cotton harvest (19.00\%) from MNH-886. The treatment $75 \mathrm{~kg} \mathrm{ha}^{-1}(26.00 \%), 75 \mathrm{~kg} \mathrm{ha}^{-1}$ + foliar (2\% TSP solution) $(27.00 \%), 100 \mathrm{~kg} \mathrm{ha}^{-1}+$ seed application (5\% solution of triple super phosphate) (27.00\%) under $100 \mathrm{~kg} \mathrm{ha}^{-1}$ phosphorous (with seed application of a 5\% solution of triple super phosphate, and foliar application of a $2 \%$ solution). $(27.00 \%)$ yielded statistically higher seed cotton harvest, the lowest seed cotton harvest was measured from control Zero kg ha-1 (19.00\%), $50 \mathrm{~kg} \mathrm{ha}^{-1}(20.00 \%), 50 \mathrm{~kg} \mathrm{ha}^{-1}$ + foliar (2\% TSP solution) (20.00\%) (Table 2). FH-Lalazar (0.02\%) showed statistically maximum ginning out turn, the lowest ginning out turn was found in two cultivars MNH-886 (0.019\%) and CIM-616 (0.018\%). Regarding the treatment under $100 \mathrm{~kg} \mathrm{ha}^{-1}$ phosphorous (with seed application of a 5\% solution of triple super phosphate, and foliar application of a $2 \%$ solution). $(0.019 \%)$ higher ginning out turn was measured, lower ginning out turn was found from control Zero $\mathrm{kg} \mathrm{ha}^{-1}(0.011 \%), 50 \mathrm{~kg} \mathrm{ha}^{-1}$ $(0.012 \%)$. Concerning cultivars and phosphorus levels interaction the highest ginning out turn $(0.028 \%)$ was recorded from FH-Lalazar with Zero $\mathrm{kg} \mathrm{ha}^{-1}$, the lowest got $(0.016 \%)$ was found from CIM-616 with Zero kg ha ${ }^{-1}$ (Table 2).

Table 2. Influence of phosphorous application methods on biological yield, seed cotton harvest, lint yield and ginning out turn of cotton cultivars

\begin{tabular}{|c|c|c|c|c|c|c|c|c|c|}
\hline Variables & Cultivars & Control & $50 \mathrm{~kg} \mathrm{ha}^{-1}$ & 50 + foliar & $75 \mathrm{~kg} \mathrm{ha}^{-1}$ & 75+foliar & $100+$ seed treat & $100+$ s+fol & Means \\
\hline \multirow{4}{*}{$\begin{array}{l}\text { Biological yield } \\
\left(\mathrm{kg} \mathrm{ha}^{-1}\right)\end{array}$} & MNH-886 & $0.56 \mathrm{fg}$ & $0.69 \mathrm{bcd}$ & $0.71 \mathrm{bcd}$ & $0.73 \mathrm{bcd}$ & $0.75 \mathrm{bcd}$ & $0.79 \mathrm{ab}$ & $0.81 \mathrm{ab}$ & $0.69 \mathrm{~b}$ \\
\hline & CIM-616 & $0.54 \mathrm{~g}$ & 0.66 def & $0.68 \mathrm{def}$ & 0.67 cde & 0.69 cde & $0.79 \mathrm{ab}$ & $0.81 \mathrm{ab}$ & $0.67 \mathrm{~b}$ \\
\hline & FH-LZ & 0.62 efg & $0.76 \mathrm{bcd}$ & $0.78 \mathrm{bcd}$ & $0.75 \mathrm{bcd}$ & $0.77 \mathrm{bcd}$ & $0.90 \mathrm{a}$ & $0.92 \mathrm{a}$ & $0.76 \mathrm{a}$ \\
\hline & & $0.57 \mathrm{c}$ & $0.70 \mathrm{~b}$ & $0.71 \mathrm{~b}$ & $0.71 \mathrm{~b}$ & $0.80 \mathrm{~b}$ & & $0.92 \mathrm{a}$ & - \\
\hline LSD (F & \multicolumn{6}{|c|}{ CULTIVAR $(C)=0.04$} & \multicolumn{3}{|c|}{$\mathrm{C} \times \mathrm{P}=0.11$} \\
\hline \multirow{4}{*}{$\begin{array}{l}\text { Seed cotton } \\
\text { harvest }(\%)\end{array}$} & MNH-886 & $17 \mathrm{fg}$ & 18 efg & 20 efg & $19 \mathrm{def}$ & $21 \mathrm{def}$ & $20 \mathrm{de}$ & $20 \mathrm{de}$ & $19 \mathrm{c}$ \\
\hline & CIM-616 & $24 \mathrm{~b}$ & 21 cde & $23 \mathrm{cde}$ & $22 \mathrm{bcd}$ & & $23 \mathrm{bc}$ & $25 \mathrm{bc}$ & $22 \mathrm{~b}$ \\
\hline & FH-LZ & $15 \mathrm{~g}$ & $19 \mathrm{df}$ & $21 \mathrm{de}$ & $37 \mathrm{a}$ & $39 \mathrm{a}$ & $38 \mathrm{a}$ & $40 \mathrm{a}$ & $27 \mathrm{a}$ \\
\hline & Means & $19 \mathrm{~b}$ & $19 \mathrm{~b}$ & $22 \mathrm{~b}$ & $26 \mathrm{a}$ & $27 \mathrm{a}$ & $27 \mathrm{a}$ & $27 \mathrm{a}$ & - \\
\hline $\operatorname{LSD}(\mathrm{P} \leq 0.05)$ & \multicolumn{6}{|c|}{ CULTIVAR $(C)=104.88$} & \multicolumn{3}{|c|}{$\mathrm{C} \times \mathrm{P}=302.7$} \\
\hline \multirow{4}{*}{ Lint yield $\left(\mathrm{kg} \mathrm{ha}^{-1}\right)$} & MNH-886 & $34.00 \mathrm{e}$ & $37.00 \mathrm{de}$ & $39.00 \mathrm{de}$ & $39.33 \mathrm{~d}$ & $41.33 \mathrm{~d}$ & $39.33 \mathrm{~d}$ & $41.33 \mathrm{~d}$ & $37.33 \mathrm{c}$ \\
\hline & CIM-616 & $40.66 \mathrm{~cd}$ & $46.00 \mathrm{~b}$ & $48.00 \mathrm{~b}$ & $44.66 \mathrm{~cd}$ & $46.66 \mathrm{~cd}$ & $40.66 \mathrm{~cd}$ & $44.66 \mathrm{~cd}$ & $42.16 \mathrm{~b}$ \\
\hline & FH-LZ & $44.66 \mathrm{~cd}$ & $48.33 \mathrm{~b}$ & $50.33 \mathrm{~b}$ & $54.00 \mathrm{a}$ & $56.00 \mathrm{a}$ & $55.33 \mathrm{a}$ & $57.33 \mathrm{a}$ & $50.58 \mathrm{a}$ \\
\hline & Means & $39.77 \mathrm{~b}$ & $43.77 \mathrm{a}$ & $43.98 \mathrm{a}$ & $44.88 \mathrm{a}$ & $44.78 \mathrm{a}$ & $45.00 \mathrm{a}$ & $45.90 \mathrm{a}$ & - \\
\hline $\operatorname{LSD}(\mathrm{P} \leq 0.05)$ & \multicolumn{6}{|c|}{ CULTIVAR $(\mathrm{C})=1.40$} & \multicolumn{3}{|c|}{$\mathrm{C} \times \mathrm{P}=4.04$} \\
\hline \multirow{4}{*}{$\begin{array}{l}\text { Ginning out turn } \\
(\%)\end{array}$} & MNH-886 & $0.019 \mathrm{~cd}$ & $0.020 \mathrm{bcd}$ & $0.050 \mathrm{bcd}$ & $0.022 \mathrm{bc}$ & $0.024 \mathrm{bc}$ & $0.018 \mathrm{~cd}$ & $0.020 \mathrm{~cd}$ & $0.019 \mathrm{ab}$ \\
\hline & CIM-616 & $0.016 \mathrm{~d}$ & $0.022 \mathrm{bc}$ & $0.024 \mathrm{bc}$ & $0.018 \mathrm{~cd}$ & $0.020 \mathrm{~cd}$ & $0.018 \mathrm{cde}$ & $/ 0.020 \mathrm{~cd}$ & $0.018 \mathrm{~b}$ \\
\hline & FH-LZ & $0.028 \mathrm{a}$ & $0.023 \mathrm{~b}$ & $0.025 \mathrm{~b}$ & $0.018 \mathrm{def}$ & $0.020 \mathrm{def}$ & $0.016 \mathrm{~d}$ & $0.020 \mathrm{~cd}$ & $0.020 \mathrm{a}$ \\
\hline & Means & $0.011 \mathrm{c}$ & $0.012 \mathrm{c}$ & $0.015 \mathrm{~b}$ & $0.017 \mathrm{~b}$ & $0.017 \mathrm{~b}$ & $0.018 \mathrm{~b}$ & $0.019 \mathrm{a}$ & - \\
\hline LSD ( & \multicolumn{6}{|c|}{ CULTIVAR $(\mathrm{C})=1.41$} & \multicolumn{3}{|c|}{$\mathrm{C} \times \mathrm{P}=4.07$} \\
\hline
\end{tabular}

Mean values sharing similar letters are statistically significant at $p=0.05$

Higher leaf area index $\left(0.65 \mathrm{~m}^{2} / \mathrm{m}^{2}\right)$ was found from CIM-616, lower leaf area index $\left(0.49 \mathrm{~m}^{2} / \mathrm{m}^{2}\right)$ was measured from MNH-886. Treatment under $100 \mathrm{~kg} \mathrm{ha}^{-1}$ phosphorous (with seed application of a 5\% solution of triple super phosphate, and foliar application of a $2 \%$ solution) $\left(0.67 \mathrm{~kg} \mathrm{~h}^{-1}\right)$ resulted the statistically maximum leaf area index, the lowest $\left(0.51 \mathrm{~m}^{2} / \mathrm{m}^{2}\right)$ was determined from control (Table 3). Cultivar FH-Lalazar $(4.79 \mathrm{~g}$ $\mathrm{m}^{-2}$ day $^{-1}$ ) gave maximum crop growth rate, the minimum was found from cultivar MNH- 
886 (4.15 $\mathrm{g} \mathrm{m}^{-2}$ day $\left.^{-1}\right)$. Concerning cultivars and phosphorus levels interaction higher crop growth rate $\left(5.12 \mathrm{~g} \mathrm{~m}^{-2}\right.$ day $\left.^{-1}\right)$ was measured from FH-Lalazar under $100 \mathrm{~kg} \mathrm{ha}^{-1}$ phosphorous (with seed application of a 5\% solution of triple super phosphate, and foliar application of a $2 \%$ solution). While the minimum $\left(4.03 \mathrm{~g} \mathrm{~m}^{-2}\right.$ day $\left.^{-1}\right)$ was calculated from cultivar MNH-886 with Zero $\mathrm{g} \mathrm{m}^{-2}$ day $^{-1}$ (Table 3). Maximum leaf area duration was calculated from FH-Lalazar (659.7), while the lowest was in MNH-886 (381.9) (Table 3). The highest phosphorus concentration was recorded in $100+$ seed treatment (591.2) and $100+$ seed + foliar (597.2). The highest phosphorus concentration was in two cultivars i.e. MNH-886 (40.08\%) and FH-LZ (45.50\%). Further detail regarding the phosphorus concentration is shown (Table 3).

Table 3. Influence of Phosphorous application methods on ginning out turn, leaf area index, crop growth rate, leaf area duration and phosphorus concentration of cotton cultivars

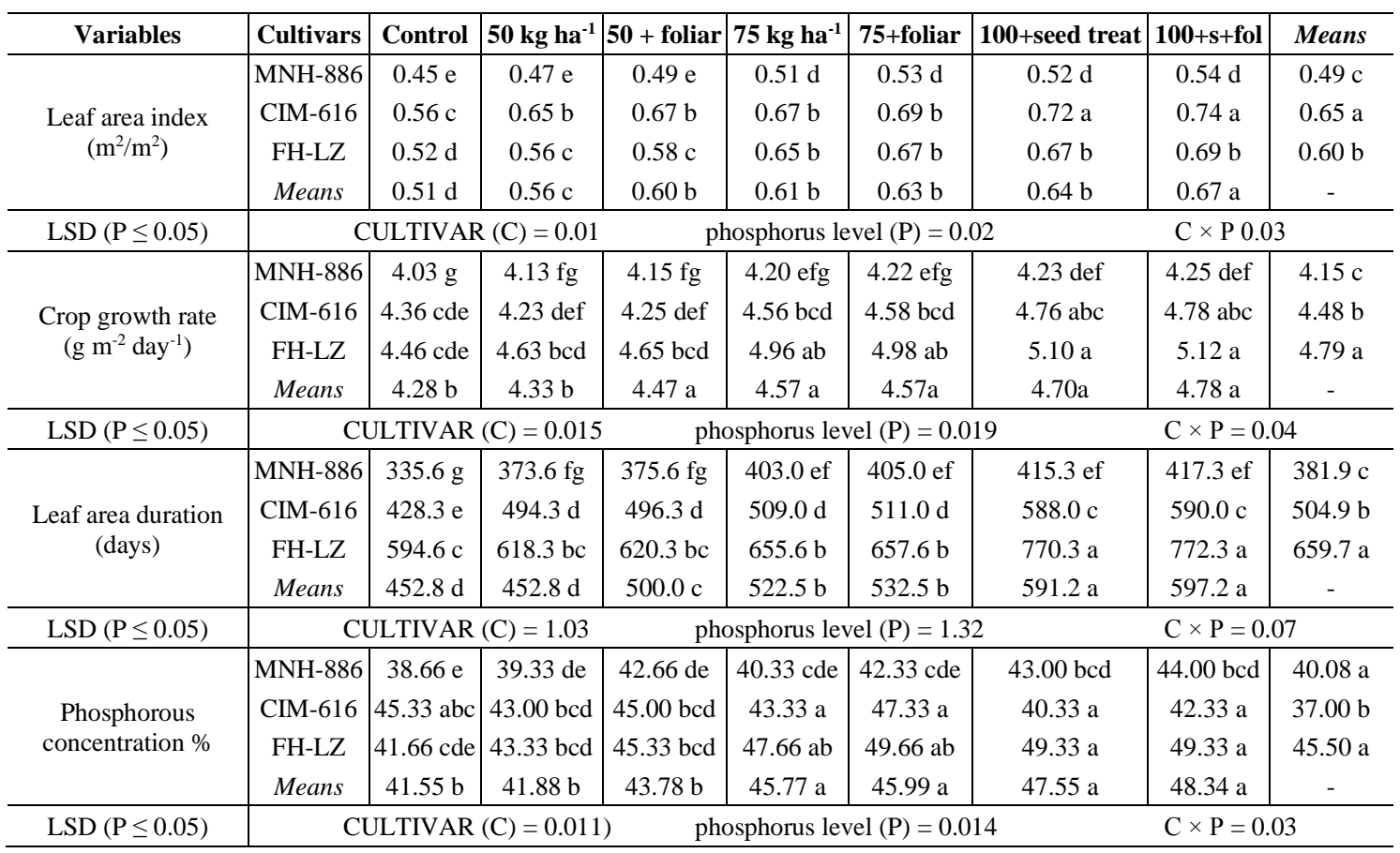

Mean values sharing similar letters are statistically significant at $p=0.05$

So due to all of this, cultivar CIM-616 (47.00), FH-Lalazar (45.50) showed statistically higher fiber uniformity. Treatment $75 \mathrm{~kg} \mathrm{ha}^{-1}$ (45.77), $75 \mathrm{~kg} \mathrm{ha}^{-1}+$ foliar (2\% TSP solution) (45.99), $100 \mathrm{~kg} \mathrm{ha}^{-1}+$ seed application (5\% solution of triple super phosphate) (47.55) under $100 \mathrm{~kg} \mathrm{ha}^{-1}$ phosphorous (with seed application of a 5\% solution of triple super phosphate, and foliar application of a $2 \%$ solution) (48.34) resulted the statistically maximum fiber uniformity, the minimum fiber uniformity was determined from control (Table 4). Cultivar CIM-616 (20.19 mm) showed maximum staple length, and cultivar MNH-886 (14.83 mm) the minimum. So, the application of level of phosphorus, treatment $75 \mathrm{~kg} \mathrm{ha}^{-1}+$ foliar (2\% TSP solution) $(91.88 \mathrm{~mm}) 100 \mathrm{~kg} \mathrm{ha}^{-1}+$ application of seed treatment (5\% TSP solution)(20.44 mm) under $100 \mathrm{~kg} \mathrm{ha}^{-1}$ phosphorous (with seed application of a 5\% solution of triple super phosphate, and foliar application of a $2 \%$ solution).(21.00 mm) gave maximum staple length, so minimum staple length was calculated from control $(15.22 \mathrm{~mm})$ and $50 \mathrm{~kg} \mathrm{ha}^{-1}(16.33 \mathrm{~mm})$ (Table 4). FH-LZ 
exhibited the highest fiber strength (30.16 tppsi) while the minimum fiber strength was determined (23.08 tppsi) from MNH-886. The treatment under $100 \mathrm{~kg} \mathrm{ha}^{-1}$ phosphorous (with seed application of a 5\% solution of triple super phosphate, and foliar application of a $2 \%$ solution) (28.00 tppsi) showed statistically higher fiber strength, lower fiber strength (24.33 tppsi) was recorded from control (Table 4). Cultivar FH-LZ showed the highest micronair (4.72 $\mu \mathrm{g} / \mathrm{inch})$, the minimum was found from cultivar MNH-886 (4.14). Application of treatment $100 \mathrm{~kg} \mathrm{ha}^{-1}+$ seed treatment (5\% TSP solution) (4.68 $\left.\mu \mathrm{g} / \mathrm{inch}\right)$ under $100 \mathrm{~kg} \mathrm{ha}^{-1}$ phosphorous (with seed application of a 5\% solution of triple super phosphate, and foliar application of a $2 \%$ solution). (4.75 $\mu \mathrm{g} / \mathrm{inch}), 75 \mathrm{~kg} \mathrm{ha}^{-1}$ (4.45 $\mu \mathrm{g} /$ inch), $75 \mathrm{~kg} \mathrm{ha}^{-1}+$ foliar (2\%TSPsolution) $(4.51 \mu \mathrm{g} / \mathrm{inch})$ resulted statistically higher micronair, lower was recorded from control (4.27 $\mu \mathrm{g} / \mathrm{inch}), 50 \mathrm{~kg} \mathrm{ha}^{-1}$ (4.34 $\mu \mathrm{g} /$ inch), 50 $\mathrm{kg} \mathrm{ha}^{-1}+5 \%$ foliar (2\% TSPsolution) $(4.40 \mu \mathrm{g} / \mathrm{inch})$. Regarding phosphorus levels and cultivars interaction the highest micronair was measured from FH-Lalazar with $100 \mathrm{~kg} \mathrm{ha}^{-}$ ${ }^{1}+$ seed treatment (5\% TSP solution) (5.06) from cultivar CIM-616 under $100 \mathrm{~kg} \mathrm{ha}^{-1}$ phosphorous (with seed application of a $5 \%$ solution of triple super phosphate, and foliar application of a $2 \%$ solution) $(5.08 \mu \mathrm{g} /$ inch $)$ (Table 4$)$.

Table 4. Influence of phosphorous application methods on fiber uniformity, staple length, fiber strength and micronair of cotton cultivars

\begin{tabular}{|c|c|c|c|c|c|c|c|c|c|}
\hline Variables & Cultivars & \begin{tabular}{|l|} 
Control \\
\end{tabular} & $50 \mathrm{~kg} \mathrm{ha}^{-1}$ & $50+$ foliar & $75 \mathrm{~kg} \mathrm{ha}^{-1}$ & 75+foliar & $100+$ seed treat & $100+$ s+fol & Means \\
\hline \multirow{4}{*}{ Fiber uniformity } & MNH-886 & $37.66 \mathrm{e}$ & $39.33 \mathrm{de}$ & $41.66 \mathrm{de}$ & 40.33 cde & 42.33 cde & $43.00 \mathrm{bcd}$ & $45.00 \mathrm{bcd}$ & $40.08 \mathrm{~b}$ \\
\hline & CIM-616 & $45.33 \mathrm{abc}$ & $43.00 \mathrm{bcd}$ & $45.00 \mathrm{bcd}$ & $49.33 \mathrm{a}$ & $49.33 \mathrm{a}$ & $50.33 \mathrm{a}$ & $52.33 \mathrm{a}$ & $47.00 \mathrm{a}$ \\
\hline & FH-LZ & 41.66 cde & $43.33 \mathrm{bcd}$ & $45.33 \mathrm{bcd}$ & $47.66 \mathrm{ab}$ & $49.66 \mathrm{ab}$ & $49.33 \mathrm{a}$ & $49.33 \mathrm{a}$ & $45.50 \mathrm{a}$ \\
\hline & Means & $41.55 \mathrm{~b}$ & $41.88 \mathrm{~b}$ & $43.78 \mathrm{~b}$ & $45.77 \mathrm{a}$ & $45.99 \mathrm{a}$ & & $48.34 \mathrm{a}$ & - \\
\hline $\operatorname{LSD}(\mathrm{P} \leq 0.05)$ & \multicolumn{3}{|c|}{ CULTIVAR $(\mathrm{C})=1.52$} & \multicolumn{4}{|c|}{ phosphorus level $(\mathrm{P})=1.93$} & \multicolumn{2}{|c|}{$\mathrm{C} \times \mathrm{P}=4.38$} \\
\hline \multirow{4}{*}{ Staple length (mm) } & MNH-886 & $13.33 \mathrm{e}$ & $14.33 \mathrm{de}$ & $16.33 \mathrm{de}$ & $14.33 \mathrm{de}$ & $16.33 \mathrm{de}$ & $17.33 \mathrm{~cd}$ & $19.33 \mathrm{~cd}$ & $14.83 \mathrm{c}$ \\
\hline & CIM-616 & $19.00 \mathrm{bc}$ & $20.33 \mathrm{abc}$ & $22.33 \mathrm{abc}$ & $21.33 \mathrm{ab}$ & $23.33 \mathrm{ab}$ & $23.00 \mathrm{a}$ & $25.00 \mathrm{a}$ & $20.91 \mathrm{a}$ \\
\hline & FH-LZ & $13.33 \mathrm{e}$ & $14.33 \mathrm{de}$ & $16.33 \mathrm{de}$ & $20.00 \mathrm{abc}$ & $22.00 \mathrm{abc}$ & $21.00 \mathrm{abc}$ & $23.00 \mathrm{abc}$ & $17.16 \mathrm{~b}$ \\
\hline & Means & $15.22 \mathrm{c}$ & $16.33 \mathrm{c}$ & $17.23 \mathrm{~b}$ & $18.55 \mathrm{~b}$ & $19.88 \mathrm{a}$ & 20 & $21.00 \mathrm{a}$ & - \\
\hline $\mathrm{LSD}(\mathrm{P} \leq 0.05)$ & \multicolumn{3}{|c|}{ CULTIVAR $(\mathrm{C})=1.30$} & \multicolumn{4}{|c|}{ phosphorus level $(\mathrm{P})=1.66$} & \multicolumn{2}{|c|}{$\mathrm{C} \times \mathrm{P}=3.76$} \\
\hline \multirow{4}{*}{ Fiber strength (tppsi) } & MNH-886 & $22.33 \mathrm{e}$ & $23.33 \mathrm{de}$ & $25.33 \mathrm{de}$ & $23.33 \mathrm{de}$ & $25.33 \mathrm{de}$ & $23.66 \mathrm{de}$ & $25.66 \mathrm{de}$ & $23.16 \mathrm{c}$ \\
\hline & CIM-616 & 2466 de & 25.00 cde & $27.00 \mathrm{cde}$ & $26.33 \mathrm{bcd}$ & $28.33 \mathrm{bcd}$ & $27.00 \mathrm{cb}$ & $29.00 \mathrm{bc}$ & $25.75 \mathrm{~b}$ \\
\hline & FH-LZ & $26.33 \mathrm{bcd}$ & $28.68 \mathrm{~b}$ & $30.68 \mathrm{~b}$ & $32.33 \mathrm{a}$ & $34.33 \mathrm{a}$ & $33.33 \mathrm{a}$ & $35.33 \mathrm{a}$ & $30.16 \mathrm{a}$ \\
\hline & Means & $24.44 \mathrm{~b}$ & $25.66 \mathrm{~b}$ & $26.00 \mathrm{~b}$ & $27.33 \mathrm{a}$ & $27.83 \mathrm{a}$ & $28.00 \mathrm{a}$ & $28.90 \mathrm{a}$ & - \\
\hline $\operatorname{LSD}(\mathrm{P} \leq 0.05)$ & \multicolumn{3}{|c|}{ CULTIVAR $(\mathrm{C})=1.15$} & \multicolumn{4}{|c|}{ phosphorus level $(\mathrm{P})=1.47$} & \multicolumn{2}{|c|}{$\mathrm{C} \times \mathrm{P}=3.32$} \\
\hline \multirow{4}{*}{ Micronair ( $\mu \mathrm{g} / \mathrm{inch})$} & MNH-886 & $4.03 \mathrm{~g}$ & $4.13 \mathrm{fg}$ & $4.15 \mathrm{fg}$ & 4.20 efg & 4.22 efg & $4.23 \mathrm{def}$ & $4.25 \mathrm{def}$ & $4.15 \mathrm{c}$ \\
\hline & CIM-616 & $4.36 \mathrm{cde}$ & $4.23 \mathrm{def}$ & 4.25 def & $4.56 \mathrm{bcd}$ & $4.58 \mathrm{bcd}$ & $4.76 \mathrm{abc}$ & $4.78 \mathrm{abc}$ & $4.48 \mathrm{~b}$ \\
\hline & FH-LZ & $4.46 \mathrm{cde}$ & $4.63 \mathrm{bcd}$ & $4.65 \mathrm{bcd}$ & $4.96 \mathrm{ab}$ & $4.98 \mathrm{ab}$ & $5.10 \mathrm{a}$ & $5.12 \mathrm{a}$ & $4.79 \mathrm{a}$ \\
\hline & Means & $4.28 \mathrm{~b}$ & $4.33 \mathrm{~b}$ & $4.47 \mathrm{a}$ & $4.57 \mathrm{a}$ & $4.57 \mathrm{a}$ & $4.70 \mathrm{a}$ & $4.78 \mathrm{a}$ & - \\
\hline $\operatorname{LSD}(\mathrm{P} \leq 0.05)$ & \multicolumn{3}{|c|}{ CULTIVAR $(\mathrm{C})=0.13$} & \multicolumn{4}{|c|}{ phosphorus level $(\mathrm{P})=0.17$} & \multicolumn{2}{|c|}{$\mathrm{C} \times \mathrm{P}=0.40$} \\
\hline
\end{tabular}

Mean values sharing similar letters are statistically significant at $p=0.05$

Pearson's correlation of various cotton traits cultivars showed that plant height shared a direct significant correlation with all other studied traits i.e. no. of bolls/plant, boll weight, yield of cotton, lint yield, biological yield, seed cotton harvest, got, LAI, CGR, LAD, phosphorus concentration, fiber uniformity, staple length, fiber strength and micronair. Moreover, it has been studied that seed cotton harvest trait gave nonsignificant correlation with lint yield. Similarly, leaf area duration gave non-significant correlation with lint yield among all the studied traits (Table 5). Correlation matrix is an 
important tool for the evaluation of trait association with each other (Anjum et al., 2018). Economic analysis of cost benefit ratio presented that maximum output can be attained with low input cost. And the highest benefit cost ratio was found 1.73 while the lowest was 1.27 during 2015, therefore in 2016 the highest was 1.81 and the lowest was 1.34 (Table 6). Eco-metrological data had long term effects on benefit cost ratio and net returns, therefore all these parameters must be calculated (Table 7).

Table 5. Pearson's correlation of different attributes of cotton cultivars

\begin{tabular}{|c|c|c|c|c|c|c|c|c|c|c|c|c|c|c|c|c|}
\hline$\frac{\frac{n}{0}}{2}$ & 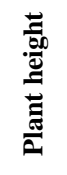 & 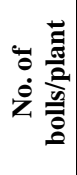 & $\begin{array}{l}\vec{E} \\
\frac{000}{20} \\
\overline{0} \\
\overline{0}\end{array}$ & 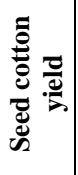 & 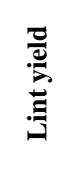 & 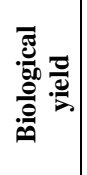 & 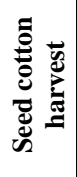 & 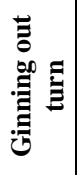 & 莺 & 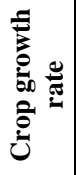 & 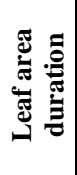 & 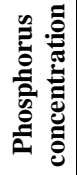 & 离 & $\begin{array}{l}\frac{5}{50} \\
\frac{0}{0} \\
\frac{0}{\bar{D}} \\
\frac{0}{D}\end{array}$ & 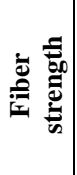 & 营 \\
\hline $\begin{array}{l}\text { Plant } \\
\text { height }\end{array}$ & 1 & & & & & & & & & & & & & & & \\
\hline $\begin{array}{c}\text { No. of } \\
\text { bolls/plant }\end{array}$ & 0.97 & 1 & & & & & & & & & & & & & & \\
\hline $\begin{array}{c}\text { Boll } \\
\text { weight }\end{array}$ & 0.93 & 0.89 & 1 & & & & & & & & & & & & & \\
\hline $\begin{array}{c}\text { Seed cotton } \\
\text { yield }\end{array}$ & 0.92 & 0.93 & 0.79 & 1 & & & & & & & & & & & & \\
\hline $\begin{array}{l}\text { Lint } \\
\text { yield }\end{array}$ & 0.91 & 0.86 & 0.99 & 0.75 & 1 & & & & & & & & & & & \\
\hline $\begin{array}{l}\text { Biological } \\
\text { yield }\end{array}$ & 0.95 & 0.95 & 0.91 & 0.81 & 0.88 & 1 & & & & & & & & & & \\
\hline $\begin{array}{c}\text { Seed cotton } \\
\text { harvest }\end{array}$ & 0.90 & 0.92 & 0.81 & 0.96 & 0.78 & 0.81 & 1 & & & & & & & & & \\
\hline $\begin{array}{l}\text { Ginning out } \\
\text { turn }\end{array}$ & 0.92 & 0.95 & 0.80 & 0.98 & 0.76 & 0.861 & 0.98 & 1 & & & & & & & & \\
\hline $\begin{array}{l}\text { Leaf area } \\
\text { index }\end{array}$ & 0.96 & 0.95 & 0.95 & 0.86 & 0.93 & 0.95 & 0.91 & 0.91 & 1 & & & & & & & \\
\hline $\begin{array}{l}\text { Crop growth } \\
\text { rate }\end{array}$ & 0.96 & 0.93 & 0.86 & 0.91 & 0.83 & 0.92 & 0.93 & 0.95 & 0.96 & 1 & & & & & & \\
\hline $\begin{array}{c}\text { Leaf area } \\
\text { duration }\end{array}$ & 0.94 & 0.92 & 0.79 & 0.90 & 0.75 & 0.90 & 0.91 & 0.93 & 0.92 & 0.99 & 1 & & & & & \\
\hline $\begin{array}{l}\text { Phosphorus } \\
\text { concentration }\end{array}$ & 0.96 & 0.94 & 0.84 & 0.95 & 0.81 & 0.90 & 0.96 & 0.97 & 0.95 & 0.99 & 0.98 & 1 & & & & \\
\hline $\begin{array}{c}\text { Fiber } \\
\text { uniformity }\end{array}$ & 0.96 & 0.94 & 0.84 & 0.95 & 0.81 & 0.90 & 0.96 & 0.97 & 0.95 & 0.99 & 0.98 & 0.99 & 1 & & & \\
\hline $\begin{array}{l}\text { Staple } \\
\text { length }\end{array}$ & 0.97 & 0.99 & 0.88 & 0.94 & 0.84 & 0.94 & 0.95 & 0.97 & 0.96 & 0.97 & 0.96 & 0.98 & 0.98 & 1 & & \\
\hline $\begin{array}{c}\text { Fiber } \\
\text { strength }\end{array}$ & 0.97 & 0.98 & 0.92 & 0.94 & 0.89 & 0.95 & 0.94 & 0.97 & 0.97 & 0.97 & 0.93 & 0.97 & 0.97 & 0.90 & 1 & \\
\hline Micronair & 0.96 & 0.93 & 0.86 & 0.91 & 0.83 & 0.92 & 0.93 & 0.95 & 0.96 & 0.99 & 0.98 & 0.99 & 0.99 & 0.97 & 0.97 & 1 \\
\hline
\end{tabular}

Values in bold are different from 0 with a significance level $\alpha=0.05$

\section{Discussion}

Different parameters such as structure of plants, production of total dry mater and economic yield become used to evaluate useful doses of fertilizer. When the main node stem was enhanced then it would be mainly obligate for height of plant, no. of bolls/plant and boll weight. Cultivar MNH-147 gave the highest height of plant and no. of bolls/plant in comparison to CIM-240. The reason was due to the growth habit of cultivar MNH-147 because it is more indeterminate as compared to CIM-240 (Malik et al., 2006). The 
performance of cultivars may varied under different concentrations of phosphorus (Ramya and Hebsur, 2018). Phosphorous is an essential nutrient, the effect was stagnant to cotton crop, enhancing no. of flowers (Russell, 2001). Yield of cotton and lint significant were enhanced through application of phosphorus which is excellent trait for higher production. It has been noted that phosphorus affects photosynthesis so as essential commodities, while these commodities are the main factors affecting the yield of cotton. Phosphorous fertilizer is the main component to give power for plant growth and enhance weight of boll in cotton. Biological yield is equivalent to the above ground biomass. It is cumulative concerning both plant weight and root weight. Biological yield plays an imperative role in the increase of cotton production. Biological yield affected different parameters viz, environment, soil, and other plant factors. Results of the current experiment demonstrated the highest biological yield (Ramya and Hebsur, 2018). Biological yield was found highest when the spacing of plants was increased (Al-Dalain et al., 2012). Leaf area index is a main parameter for crop growth. It also describes raw materials produced by total radiation to plant for the initiation of leaf. There would be different ranges of leaf area index in young cotton plant. The cotton plant has ability to capture all of solar radiation when leaf area index reached the range of 3 (Zhou et al., 2011). Crop growth rate would be measured on different strategies such as that maximum calculated from NIAB-846 lower doses of nitrogen $\left(50 \mathrm{~kg} \mathrm{ha}^{-1}\right)$, the best crop growth rate on next cultivars, CIM-496, NIAB-824, demonstrated with increase of nitrogen doses of $100,150 \mathrm{~kg}$ nitrogen per hectare simultaneously. While the reduction of CGR was observed with high stress treatments such as moisture stress and stages of vegetative growth (Hassan et al., 2016). Dry matter production of a crop is dependent on leaf area duration. Alkaline soils gave the highest yield when different relationship occurred between leaf area duration, yield and biomass. When there was no competition of weed, it was observed that leaf area duration gave the highest (136.85 days) in plots after transplantation so the reduction occurred due to the increase of competition. Reductions of LAD was observed in plots with competition of barnyard grass throughout the crop growth season. (Chen et al., 2018; Verma et al., 2018).

Table 6. Influence of phosphorus foliar application on benefit cost ratio (BCR) of cotton

\begin{tabular}{|c|c|c|c|c|c|c|c|}
\hline Treatments (foliar applications) 2015 & $\begin{array}{c}\text { Yield } \\
\left(\mathrm{kg} \mathrm{ha}^{-1}\right)\end{array}$ & $\begin{array}{c}\text { Value } \\
\left(\$ \text { ha }^{-1}\right)\end{array}$ & $\begin{array}{c}\text { Stick value } \\
\left(\$ \mathbf{h a}^{-1}\right)\end{array}$ & $\begin{array}{c}\text { Gross value } \\
\left(\$ \mathrm{ha}^{-1}\right)\end{array}$ & $\begin{array}{c}\text { Total cost } \\
\left(\$ \mathbf{h a}^{-1}\right)\end{array}$ & $\begin{array}{c}\text { Net return } \\
\left(\$ \mathrm{ha}^{-1}\right)\end{array}$ & BCR \\
\hline Po $=$ Control & 1941.1 & 908.8763 & 53.51171 & 962.388 & 754.1806 & 208.2074 & 1.27 \\
\hline $\mathrm{P}_{1}=50 \mathrm{~kg} / \mathrm{ha}$ & 1982.3 & 928.1672 & 53.51171 & 981.6789 & 755.5184 & 226.1605 & 1.29 \\
\hline $\mathrm{P}_{2}=50+$ & 2297.2 & 1075.612 & 53.51171 & 1129.124 & 778.9298 & 350.194 & 1.44 \\
\hline $\mathrm{P}_{3}=75 \mathrm{~kg} / \mathrm{ha}$ & 2639.0 & 1235.652 & 66.88963 & 1302.542 & 785.9532 & 516.5886 & 1.65 \\
\hline $\mathrm{P}_{4}=75+$ & 2798 & 1310.1 & 66.88963 & 1376.99 & 794.3144 & 582.6756 & 1.73 \\
\hline $\mathrm{P}_{5}=100 \mathrm{~kg} / \mathrm{ha}$ & 2798 & 1310.1 & 66.88963 & 1376.99 & 794.3144 & 582.6756 & 1.73 \\
\hline$P_{6}=100+$ & 2763 & 1293.712 & 66.88963 & 1360.602 & 794.6488 & 565.9532 & 1.71 \\
\hline Treatments (foliar applications) 2016 & $\begin{array}{l}\text { Yield } \\
\left(\$ \text { ha }^{-1}\right)\end{array}$ & $\begin{array}{l}\text { Value } \\
\left(\$ \text { ha }^{-1}\right)\end{array}$ & $\begin{array}{l}\text { Stick value } \\
\left(\$ \text { ha }^{-1}\right)\end{array}$ & $\begin{array}{c}\text { Gross value } \\
\left(\$ \mathbf{h a}^{-1}\right)\end{array}$ & $\begin{array}{c}\text { Total cost } \\
\left(\$ \text { ha }^{-1}\right)\end{array}$ & $\begin{array}{l}\text { Net return } \\
\quad\left(\$ \mathbf{h a}^{-1}\right)\end{array}$ & BCR \\
\hline Po $=$ Control & 1941.1 & 973.796 & 53.51171 & 1027.308 & 762.2074 & 265.1003 & 1.34 \\
\hline$P_{1}=50 \mathrm{~kg} / \mathrm{ha}$ & 2002.3 & 1004.498 & 53.51171 & 1058.01 & 781.9398 & 276.0702 & 1.35 \\
\hline $\mathrm{P}_{2}=50+$ & 2004.3 & 1005.502 & 53.51171 & 1059.013 & 782.2742 & 276.7391 & 1.35 \\
\hline $\mathrm{P}_{3}=75 \mathrm{~kg} / \mathrm{ha}$ & 2638.0 & 1323.411 & 66.88963 & 1390.301 & 792.9766 & 597.3244 & 1.75 \\
\hline$P_{4}=75+$ & 2651.0 & 1329.933 & 66.88963 & 1396.823 & 793.311 & 603.5117 & 1.76 \\
\hline $\mathrm{P}_{5}=100 \mathrm{~kg} / \mathrm{ha}$ & 2763.9 & 1386.572 & 66.88963 & 1453.462 & 802.3411 & 651.1204 & 1.81 \\
\hline $\mathrm{P}_{6}=100+$ & 2769.9 & 1389.582 & 66.88963 & 1456.472 & 802.6756 & 653.796 & 1.81 \\
\hline
\end{tabular}


Table 7. Eco-meteorological data of Multan, Punjab (Pakistan)

\begin{tabular}{|c|c|c|c|c|c|c|c|c|c|c|c|c|c|c|}
\hline \multirow{3}{*}{ Months } & \multicolumn{4}{|c|}{ Temperature (C) } & \multicolumn{4}{|c|}{ Relative humidity (\%) } & \multirow{2}{*}{\multicolumn{2}{|c|}{$\begin{array}{c}\text { Wind speed } \\
\left(\mathbf{k m ~ h}^{-1}\right)\end{array}$}} & \multirow{2}{*}{\multicolumn{2}{|c|}{$\begin{array}{l}\text { Sunshine } \\
\text { hours (h) }\end{array}$}} & \multirow{2}{*}{\multicolumn{2}{|c|}{$\begin{array}{c}\text { Rainfall } \\
(\mathbf{m m})\end{array}$}} \\
\hline & Max. & Min. & Max. & Min. & $8 \mathrm{AM}$ & 5 PM & 8 AM & 5 PM & & & & & & \\
\hline & \multicolumn{2}{|c|}{2015} & \multicolumn{2}{|c|}{2016} & \multicolumn{2}{|c|}{2015} & \multicolumn{2}{|c|}{2016} & 2015 & 2016 & 2015 & 2016 & 2015 & 2016 \\
\hline June & 38.7 & 29.1 & 39.8 & 30.4 & 71.4 & 64.1 & 62.1 & 42.4 & 7.9 & 7.7 & 7.7 & 7.5 & 50.6 & 1.3 \\
\hline July & 38.0 & 29.7 & 36.7 & 29.3 & 71.7 & 57.1 & 70.6 & 51.5 & 6.7 & 7.3 & 7.8 & 6.5 & 40.0 & 51.5 \\
\hline August & 35.1 & 27.8 & 35.6 & 28.3 & 77.0 & 67.2 & 80.9 & 61.5 & 5.4 & 6.6 & 7.0 & 8.7 & 74.2 & 16.5 \\
\hline September & 35.1 & 25.3 & 34.0 & 25.5 & 79.4 & 63.7 & 85.4 & 65.5 & 4.9 & 4.1 & 8.6 & 7.3 & 0.0 & 4.2 \\
\hline October & 35.1 & 24.3 & 31.4 & 20.4 & 77.8 & 64.6 & 83.1 & 63.3 & 3.3 & 2.6 & 7.7 & 6.0 & 3.2 & 17.6 \\
\hline November & 26.8 & 13.0 & 26.2 & 11.9 & 84.0 & 74.5 & 86.0 & 68.0 & 2.5 & 2.0 & 5.6 & 5.0 & 0.0 & 0.0 \\
\hline December & 20.3 & 9.2 & 17.7 & 6.8 & 89.6 & 75.0 & 93.6 & 74.9 & 3.0 & 2.5 & 4.8 & 3.7 & 0.0 & 0.0 \\
\hline
\end{tabular}

Many researcher papers demonstrated that phosphorous gave response to cotton crop such as that (Magare et al., 2018; Rosace et al., 2018). Khan et al., (2014) demonstrated FU gave a positive response for Ginning out turn and value of micronair. Positive response of fiber uniformity was found on yield of cotton for fiber strength whereas negative response demonstrated for mean length and upper half mean length. Fiber uniformity, staple length, fiber strength and micronair are excellent traits to enhance the potential of textile industry. All these traits were probably enhanced with phosphorus application.

Cotton growers have many difficulties due to enhanced commodities. So, increase of inputs cost resulting in decrease of output/ profit.. Improper fertilizer is a main source to increase the production cost. Most of all growers of cotton use nitrogen fertilizer; while $85 \%$ of growers use phosphorous fertilizer for higher rate of production (Miao et al., 2018). Fertilizer cost is a major problem and has the $2^{\text {nd }}$ largest commodity value and accounts for $22 \%$ of total cost production (Makhdum et al., 2001). Phosphorous is a main nutrient but overall in cotton crop the efficiency of phosphorous is low because farmer could not facilitate phosphorous fertilizer in all soil type. Due to all of this various research studies on cotton which responds to phosphorus positively and economically were conducted (Miao et al., 2018). Results demonstrated that phosphorus is a main nutrient for cotton crop for the highest yield of cotton compared to nitrogen. So, phosphorus provides positive response for better production of cotton in Punjab.

\section{Conclusion}

It is concluded that decreased amount of phosphorus demonstrated in decreased yield of cotton along with its related traits. However, cultivar FH-LZ along with phosphorous level under $100 \mathrm{~kg} \mathrm{ha}^{-1}$ phosphorous (with seed application of a 5\% solution of triple super phosphate, and foliar application of a $2 \%$ solution) demonstrated the highest values and excellent performance for many yield traits.

Therefore, it is recommended that farmers and researchers of the vicinity may use exogenous phosphorus to get the highest yield and excellent quality of fiber of cotton crop. 


\section{REFERENCES}

[1] Ahmad, D., Chani, M. I., Rauf, A., Afzal, M. (2016): Economic analysis of cotton cultivation under agro-climatic conditions of district Muzaffargarh. - American Journal of Agric. \& Environ. Sci 16(8): 1498-1503.

[2] Ahmad, R., Anjum, M. A. (2018): Applications of molecular markers to assess genetic diversity in vegetable and ornamental crops. - A Review. J. Hort. Sci. Technol. 1: 1-7.

[3] Ahmad, R., Malik, W., Anjum, M. A. (2019): Genetic Diversity and Selection of Suitable Molecular Markers for Characterization of Indigenous Zizyphus Germplasm. - ErwerbsObstbau 6: 1-9.

[4] Al-dalain, S. A., Abdel-ghani, A. H., Dala'een, A. (2012): Effect of planting date and spacing on growth and yield of fennel (Foeniculum vulgare mill.) under irrigated conditions. - Pak. J. Bio. Sci. 15: 1126-1132.

[5] Anjum, M. A., Rauf, A., Bashir, M. A., Ahmad, R. (2018): The evaluation of biodiversity in some indigenous Indian jujube (Zizyphusmauritiana) germplasm through physicochemical analysis. - Acta Scient. Polo-Hortorum Cultus 17(4): 39-52.

[6] Apel, K., Hirt, H. (2004): Reactive oxygen Species: metabolism, oxidative stress, and signal transduction. - Annu. Rev. Plant Biol. 55: 373-399.

[7] Araújo, E. O., Santos, G. Q., Oliveira, M. A., Camacho, Dresch, D. M. (2012): Nutritional efficiency of cowpea varieties in the absorption of phosphorus. - Agronomía Colombiana 30: 419-424.

[8] Baloch, M. J., Khan, N. U., Rajput, M. A., Jatoi, W. A., Gul, S., Rind, I. H., Veesar, N. F. (2014): Yield related morphological measures of short duration cotton genotypes. - J. Anim. Plant Sci. 24(4): 1198-211.

[9] Bronson, K. F., Keeling, J. W., Booker, J. D., Chua, T. T., Wheeler, T. A., Boman, R. K., Lascano, R. J. (2003): Influence of landscape position, soil series and phosphorus fertilizer on cotton lint yield. - Agron. J. 95: 949-957.

[10] Bronson, K. F., Onken, A. B., Booker, J. D., Lascano, R. J., Provin, T. L., Torbert, H. A. (2001): Irrigated cotton yields as affected by phosphorus fertilizer and landscape position. - Commun. Soil Sci. Plant Anal. 32: 1959-1967.

[11] Chen, J., Liu, L., Wang, Z., Sun, H., Zhang, Y., Lu, Z., Li, C. (2018): Determining the effects of nitrogen rate on cotton root growth and distribution with soil cores and mini rhizotrons. - PloS One 13(5): e0197284.

[12] Crozier, C. R., Walls, B., Hardy, D. H., Barnes, J. S. (2004): Response of cotton to P and K soil fertility gradients in North Carolina. - J. Cotton. Sci. 8: 130-141.

[13] Dohary, C. G., Rochester, I. J., Blair, G. J. (2004): Response of field grown cotton (Gossypium hirsutum L.) to phosphorus fertilization on alkaline soils in eastern Australia. - Aust. J. Soil Res. 42: 913-920.

[14] Fageria, N. K., Moraes, M. F. (2013): Phosphorus nutrition of lowland rice in tropical lowland soil. - Communications in Soil Science and Plant Analysis 44: 2932-2940.

[15] Fageria, N. K., Baligar, V. C., Moreira, A., Portes, T. A. (2010): Dry bean genotypes evaluation for growth, yield components and phosphorus use efficiency. - J. Plant Nut. 33: 2167-2181.

[16] Gaikwad, J. L. (2018): Studies on weather indices in hirsutum cotton under varied condition. - Doctoral dissertation, Vasantrao Naik Marathwada Krishi Vidyapeeth, Parbhani.

[17] Girma, K., Roger, W., Freeman, K., Randal, K., Boman, W., Raun, R. (2007): Cotton Lint Yield and Quality As Affected by Applications of N, P and K Fertilizers Kefyalew. The J. Cotton Sci. 11: 12-19.

[18] G. O. P. (2018): Economic survey of Pakistan 2017-2018. - Ministry of Food and Agriculture, Finance Division, Economic Advisor Wing, Islamabad, Pakistan. 
[19] Hassan, M., Maqsood, M., Wajid, S. A., Ranjha, A. M. (2016): Impact of moisture stress and nitrogen on crop growth rate, nitrogen use efficiency, and harvest index of cotton (Gossypium hirsutum L.) - Pak. J. Agri. Sci. 53: 171-180.

[20] Hunt, K. H. (1978): Kinematic Geometry of Mechanisms. - Oxford University Press, Oxford.

[21] Khan, M. I., Dasti, M. A., Mahmood, Z. (2014): Effects of fiber traits on seed cotton yield of cotton (Gossypium hirsutum L.) - J. Agric. Res. 2: 52-56.

[22] Magare, P. N., Jadhao, S. D., Farkade, B. K., Mali, D. V. (2018): Effect of levels of potassium on yield, nutrient uptake, fertility status and economics of cotton grown in vertisol. - Int. J. Curr. Microbiol. App. Sci. 7: 1292-1300.

[23] Makhdum, M. I., Malik, M. N. A., Chaudhry, F. I., Shabab-Ud-Din, C. (2001): Effect of gypsum as a sulphur fertilizer in cotton production. - Int. J. Agric. Biol. Sci. 3: 298-300.

[24] Malik, M. N. A., Chaudhry, F. I., Makhdum, M. I. (2006): Investigation on phosphorus availability and seed cotton yield in silt loam soils. - J. An. Plant Sci. 6: 21-23.

[25] Miao, S., Pengcheng, L., Cangsong, Z., Shuai, L., Aizhong, L., Huimin, H., Jingran, L., Helin, D. (2018): Effects of low phosphorus stress on root morphology and physiological characteristics of different cotton genotypes at the seedling stage. - Cotton Sci. 30: 45-52.

[26] Pakistan Meteorological Department (2015-2016): Government of Pakistan. http://www.pmd.gov.pk/.

[27] Ramya, S. H., Hebsur, N. S. (2018): Dry matter production and yield of BT cotton as influenced by available phosphorus in Vertisols. - Asian J. Soil Sci. 13: 63-69.

[28] Rosace, G., Castellano, A., Trovato, V., Iacono, G., Malucelli, G. (2018): Thermal and flame retardant behaviour of cotton fabrics treated with a novel nitrogen-containing carboxyl-functionalized organophosphorus system. - Carbohyd. Poly. 196: 348-358.

[29] Russell, E. W. (2001): Soil Condition and Plant Growth. - The English Language Book Society and Longman, London, pp. 56-87.

[30] Tisarum, R., Theerawitaya, C., Samphumphung, T., Takabe, T., Cha-um, S. (2019): Exogenous foliar application of glycine betaine to alleviate water deficit tolerance in two indica rice genotypes under greenhouse conditions. - Agronomy 9(3): 138.

[31] Vance, C. P., Stone, U. C., Allan D. L. (2003): Phosphorus acquisition and use critical adaptations by plants for securing a nonrenewable resource. - New Phytologist 157: 423447.

[32] Verma, N., Chaudhary, S., Goyal, S. (2018): Long term effects of inorganic fertilizers and organic amendments on ammonification and nitrification activity of soils under cotton wheat cropping system. - Int. J. Curr. Microbiol. App. Sci 7: 718-724.

[33] Zambrosi, F. C., Junior, D. M., Furlani, R., Quaggio, J. A., Boaretto, R. M. (2012): Efficiency of phosphorus uptake and utilization in citrus rootstocks. RevistaBrasileira de Ciência do Solo. 36: 486-496.

[34] Zhang, M., Alva, A. K., Li, Y. C., Calvert, D. V. (2012): Aluminum and iron fractions affecting phosphorus solubility and reactions in selected sandy soils. - Soil Sci. 166: 940948.

[35] Zhou, X. B., Chen, Y. H., Ouyang, Z. (2011b): Row spacing effect on leaf area development, light interception, crop growth and grain yield of summer soybean crops in Northern China. - Afr. J. Agri. Res. 6: 1430-1437. 